\title{
Performed Notification Message
}

National Cancer Institute

\section{Source}

National Cancer Institute. Performed Notification Message. NCI Thesaurus. Code C93930.

The actual text that was included in the notification. 